\title{
A Coalition Formation Game for Energy-efficient Cooperative Spectrum Sensing in Cognitive Radio Networks with Multiple Channels
}

\author{
Xiaolei Hao, Man Hon Cheung, Vincent W.S. Wong, and Victor C.M. Leung \\ Department of Electrical and Computer Engineering \\ The University of British Columbia, Vancouver, Canada \\ e-mail: \{xiaoleih, mhcheung, vincentw, vleung\}@ece.ubc.ca
}

\begin{abstract}
Spectrum sensing is one of the key technologies to realize spectrum reuse and increase the spectrum efficiency in cognitive radio networks (CRNs). In this paper, we study energy-efficient cooperative multi-channel spectrum sensing in CRNs. We first propose a cooperative spectrum sensing and accessing (CSSA) scheme for all the secondary users (SUs). The SUs cooperatively sense the licensed channels of the primary users (PUs) in the sensing slot. If a channel is determined to be idle, the SUs which have sensed that channel will have a chance to transmit packets in the data transmission slot. We then formulate this multi-channel spectrum sensing problem as a coalition formation game, where a coalition corresponds to the SUs that have chosen to sense and access a particular channel. The utility function of each coalition takes into account both the sensing accuracy and energy efficiency. We propose distributed algorithms to find the optimal partition that maximizes the aggregate utility of all the coalitions in the system. We prove analytically that the proposed algorithms terminate at a stable partition that achieves the optimal aggregate utility. Simulation results show that the proposed algorithms result in the selforganization of the SUs that achieves a higher aggregate utility after each iteration. Also, the convergence and optimality of the proposed algorithms are proved by simulation results.
\end{abstract}

\section{INTRODUCTION}

Spectrum scarcity is a severe problem in wireless communications. A report released by the Federal Communications Commission (FCC) [1] suggested that the use of fixed spectrum allocation results in spectrum holes. In order to fully utilize the scarce spectrum resources, the concept of cognitive radio was proposed [2], which the unlicensed users (secondary users) are allowed to dynamically and opportunistically access the licensed bands in a way that do not cause interference to the legacy spectrum holders (primary users). In cognitive radio networks (CRNs), the secondary users (SUs) have to obtain information about the existence of the primary users (PUs) and spectrum usage by spectrum sensing [3], [4]. As for spectrum sensing, each $\mathrm{SU}$ is required to sense the radio environment within its operating range to find the licensed spectrum which is not occupied by the PUs in order to enable dynamic spectrum access in CRNs.

In the design of spectrum sensing algorithms, both sensing accuracy and energy efficiency are important design criteria. In [5], Liang et al. studied the problem of optimizing the sensing duration to maximize the achievable throughput for the secondary network under the constraint of the probability of detection. In [6], Saad et al. studied the cooperative incentives of the SUs that seek to cooperate with each other to improve the detection performance. The collaborative spectrum sensing problem was modeled as a coalitional game, and distributed algorithms were proposed. In [7], $\mathrm{Su}$ et al. proposed a spectrum sensing scheme for SUs to reduce the energy consumption in spectrum sensing while guaranteeing the priority of the PU and the spectrum usage time of the SUs. In [8], Song et al. focused on multi-channel coordination in cooperative spectrum sensing. They studied the theoretical improvement made by multi-channel coordination and derived a theoretical performance bound. In [9], Zhao et al. proposed a slotted transmission protocol for SUs using a periodic sensing strategy with optimal dynamic access. An optimal spectrum sensing framework was proposed in [10] to solve both the interference avoidance and the spectrum efficiency problems. In [11], Wang et al. proposed a distributed cooperative multichannel spectrum sensing scheme for the non-infrastructure based CRNs. The SUs with the same selected channel form coalitions through coalitional game.

In general, there are multiple channels in a CRN. However, the works in [5]-[7] considered the simple case, where there is only one licensed channel over the spectrum of interest. The works in [5], [6], [8], [9], [11] considered only channel utilization and sensing accuracy, and ignored the energy efficiency in their design. Moreover, the system models in [7], [9] focus on local spectrum sensing, which is susceptible to sensing problems due to noise uncertainty, fading, shadowing, and hidden PUs [3]. It has been shown that the sensing performance can be improved significantly if cooperative spectrum sensing is used [12], [13].

In this paper, we study energy-efficient cooperative multichannel spectrum sensing in CRNs. We consider the setting with multiple licensed frequency channels and multiple SUs. We assume that each SU first chooses one licensed channel to sense locally. Then all the SUs that choose to sense the same channel cooperatively determine if the channel is busy or idle. If it is determined to be idle, one of SUs which has sensed that channel can access it. We consider both the spectrum sensing accuracy and energy efficiency in our model, and analyze the 


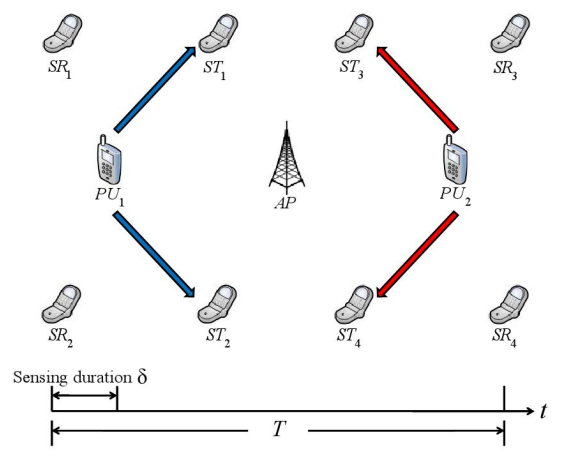

(a) Sensing slot

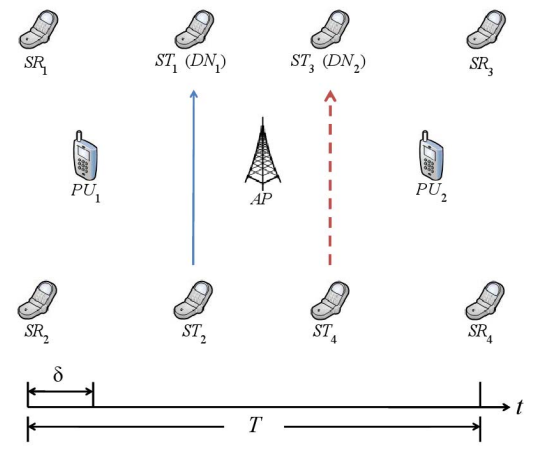

(b) Sensing decision

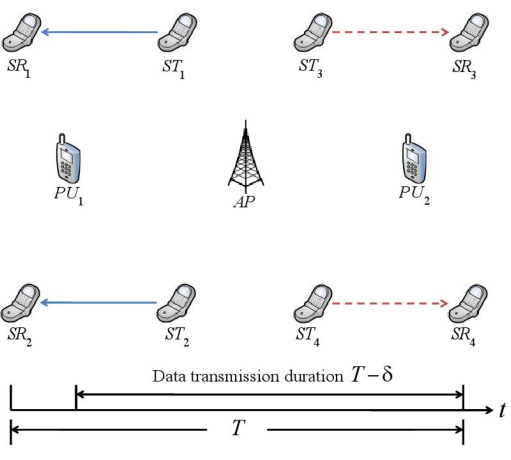

(c) Data transmission slot

Fig. 1. An example of our system model with $M=2$ and $N=4$, where the blue and red colours denote the operations in channels 1 and 2, respectively. (a) Each ST senses one licensed channel during the sensing slot. (b) When the sensing time $\delta$ expires, each ST sends its sensing result to one of the STs in that channel that acts as a DN. (c) If the channel is determined to be idle by the DN, one of the STs in that channel can transmit data to the corresponding secondary receiver in the data transmission slot.

behavior of the SUs by using coalition formation game [14]. To the best of our knowledge, this work is the first paper that applies coalition formation game to study energy-efficient cooperative multi-channel spectrum sensing in CRNs.

The contributions of our work are summarized as follows:

- We study energy-efficient cooperative spectrum sensing for the SUs in a CRN, where there are multiple licensed frequency channels. We propose a cooperative spectrum sensing and accessing (CSSA) scheme for the SUs.

- We formulate the spectrum sensing problem as a coalition formation game, where the utility function of each coalition takes into account both the sensing accuracy and energy efficiency.

- In order to maximize the aggregate utility of all the coalitions in the system, we propose distributed algorithms for the CSSA scheme. We prove analytically that they terminate at a stable partition that achieves the optimal aggregate utility of the system.

- Simulation results show that our proposed CSSA scheme achieves a better performance than the noncooperative spectrum sensing and accessing (NSSA) scheme in terms of aggregate utility. Besides, the results show that a higher aggregate utility is achieved by the proposed algorithms after each iteration, and there is a stable partition.

This paper is organized as follows. Section II describes the system model and the CSSA scheme. The coalition formation game analysis is presented in Section III. Section IV presents the simulation results. Conclusions are given in Section V.

\section{System Model AND CSSA Scheme}

As shown in Figure 1, we consider a CRN with one access point (AP), $M$ PUs, and $N$ secondary transmitter-receiver (ST$\mathrm{SR}$ ) pairs. In this paper, since the secondary transmitter (ST) is responsible for spectrum sensing, we use the term ST and SU interchangeably. Let $\mathcal{M}=\{1, \ldots, M\}$ be the set of PUs and $\mathcal{N}=\{1, \ldots, N\}$ be the set of STs. For simplicity, we assume that each PU has its own licensed channel with bandwidth $B$. Thus, there are $M$ non-overlapped licensed frequency bands in total. The $M$ PUs are sending primary data to the AP. The $N$ ST-SR pairs are located in the same area as the PUs, where $S T_{j}, \forall j \in \mathcal{N}$ seeks to exploit possible transmission opportunities in the $M$ licensed frequency bands of the PUs. We assume that each $S T_{j}$ always has data to send and no traffic requirement is imposed on the STs. In other words, $S T_{j}$ transmits data in a best-effort manner.

We consider the frame structure designed for periodic spectrum sensing, where each frame consists of one sensing slot and one data transmission slot as shown in the bottom part in Figure 1. We use $T$ to denote the frame duration. We assume that all SUs have the same spectrum sensing duration, and we use $\delta(0<\delta \leq T)$ to denote the spectrum sensing time of the SUs. Therefore, the data transmission duration is $T-\delta$. We assume that the received signal of the PUs is sampled at sampling frequency $f_{s}$ at $S T_{j}, \forall j \in \mathcal{N}$. In addition, $\delta$ is a multiple of $1 / f_{s}$. Thus, the number of samples is $\delta f_{s}$, which is an integer. We also assume that $T$ is a multiple of $1 / f_{s}$.

Let $\mathcal{H}_{1, i}$ and $\mathcal{H}_{0, i}$ be the hypothesis that $P U_{i}, i \in \mathcal{M}$ is active and inactive, respectively. For $j \in \mathcal{N}, S T_{j}$ can perform spectrum sensing by itself in the licensed frequency band of $P U_{i}, \forall i \in \mathcal{M}$ and determines the probabilities of detection and false alarm. The probability of detection is the probability of correctly detecting the appearance of $P U_{i}$ under hypothesis $\mathcal{H}_{1, i}$ (i.e., a busy channel is determined to be busy correctly). The probability of false alarm is the probability of falsely declaring the appearance of the primary signal under hypothesis $\mathcal{H}_{0, i}$ (i.e., an idle channel is determined to be busy).

We assume the noise is a Gaussian, independent and identically distributed (iid) random process with mean zero and variance $\sigma_{n}^{2}$. The primary signal of $P U_{i}, i \in \mathcal{M}$ received by $S T_{j}, \forall j \in \mathcal{N}$ is an iid random process with the same mean zero and variance $\sigma_{s, i, j}^{2}$. The primary signal is independent of the other primary signals and the noise. We denote $\gamma_{i, j}=\sigma_{s, i, j}^{2} / \sigma_{n}^{2}$ as the received signal-to-noise ratio (SNR) of $P U_{i}, i \in \mathcal{M}$ measured at $S T_{j}, j \in \mathcal{N}$ under the hypothesis $\mathcal{H}_{1, i}$. We use $\varepsilon$ to denote the detection threshold for all the $\mathrm{STs}$. We consider the circularly symmetric complex Gaussian 
(CSCG) noise case, and we also assume that the primary signal is complex phase shift keying (PSK) modulated signal. With the use of energy detection [3], [15], under hypothesis $\mathcal{H}_{0, i}$, the probability of false alarm in channel $i \in \mathcal{M}$ by $S T_{j}$ is given by [5]

$$
P_{f, i, j}(\delta)=Q\left(\left(\frac{\varepsilon}{\sigma_{n}^{2}}-1\right) \sqrt{\delta f_{s}}\right),
$$

where $Q$ is the complementary distribution function of the standard Gaussian. Besides, under hypothesis $\mathcal{H}_{1, i}$, the probability of detection in channel $i \in \mathcal{M}$ by $S T_{j}, j \in \mathcal{N}$ is

$$
P_{d, i, j}(\delta)=Q\left(\left(\frac{\varepsilon}{\sigma_{n}^{2}}-\gamma_{i, j}-1\right) \sqrt{\frac{\delta f_{s}}{2 \gamma_{i, j}+1}}\right) .
$$

It has been reported that the hidden node problem, deep fading and shadowing degrade the performance of local spectrum sensing of individual SU [3], [16]. To overcome this problem, cooperative spectrum sensing was proposed, where SUs share information and combine results from independent measurements. In our system model, we assume that each SU chooses to sense a licensed frequency band independently, and sends its spectrum sensing result to a decision node (DN) for that channel when the sensing time $\delta$ expires. Among all $S T_{j}, j \in \mathcal{N}$ which choose to sense channel $i \in \mathcal{M}, D N_{i}$ is selected according to Algorithm 1, which will be presented in Section III. The $D N_{i}$ will combine the sensing results of the SUs which choose to sense channel $i$ and determine the status (i.e., busy or idle) of channel $i$. We consider an example shown in Figure 1, where there are two licensed channels and four ST-SR pairs. At the beginning of each frame, as shown in Figure 1(a), $S T_{1}$ and $S T_{2}$ in channel 1 start sensing the licensed channel of $P U_{1}, S T_{3}$ and $S T_{4}$ in channel 2 start sensing the licensed channel of $P U_{2}$. In Figure 1(b), $S T_{1}$ and $S T_{3}$ work as the $D N_{1}$ and $D N_{2}$, respectively. Each $\mathrm{SU}$ will send its spectrum sensing decision to the corresponding $D N_{i}$ when its sensing time $\delta$ expires. The $D N_{i}$ will make the final spectrum sensing decision for the licensed channel $i \in \mathcal{M}$.

We assume that the DN decides on the channel status based on a decision fusion rule to combine the sensing results of the SUs [5]. For simplicity, we assume that $O R$ rule is used. That is, if one of the STs reports that there is an active PU, then the final decision declares that the channel is busy. We assume that the decisions made by each ST in the same channel are independent. Let $\mathcal{S}_{i}$ be the set of STs that choose to sense and access channel $i$. We have $\mathcal{S}_{i} \subseteq \mathcal{N}, \forall i \in \mathcal{M}$ and $\bigcup_{i \in \mathcal{M}} \mathcal{S}_{i}=$ $\mathcal{N}$. Moreover, since we assume that $S T_{j}, \forall j \in \mathcal{N}$ can choose to sense only one channel in each frame, we have $\mathcal{S}_{i} \cap \mathcal{S}_{l}=$ $\emptyset, \forall i, l \in \mathcal{M}, i \neq l$. The probability of false alarm $P_{f, i}$ under the hypothesis $\mathcal{H}_{0, i}$ and the probability of detection $P_{d, i}$ under the hypothesis $\mathcal{H}_{1, i}$ in channel $i, i \in \mathcal{M}$ are given by

$$
\begin{aligned}
& P_{f, i}(\delta)=1-\prod_{j \in \mathcal{S}_{i}}\left(1-P_{f, i, j}(\delta)\right), \\
& P_{d, i}(\delta)=1-\prod_{j \in \mathcal{S}_{i}}\left(1-P_{d, i, j}(\delta)\right) .
\end{aligned}
$$

For the licensed frequency band $i \in \mathcal{M}$, we define $P_{\mathcal{H}_{1, i}}$ as the probability that $P U_{i}$ is active, and $P_{\mathcal{H}_{0, i}}$ as the probability that $P U_{i}$ is silent. Therefore, we have $P_{\mathcal{H}_{1, i}}+P_{\mathcal{H}_{0, i}}=1$. Let $P_{0 \mid 0, i}$ be the probability that $P U_{i}$ is silent and the decision made by $D N_{i}$ is not a false alarm. It is given by

$$
P_{0 \mid 0, i}(\delta)=P_{\mathcal{H}_{0, i}}\left(1-P_{f, i}(\delta)\right)
$$

Let $P_{0 \mid 1, i}$ be the probability that $P U_{i}$ is active but $D N_{i}$ fails to detect the presence of the primary signal. It is given by

$$
P_{0 \mid 1, i}(\delta)=P_{\mathcal{H}_{1, i}}\left(1-P_{d, i}(\delta)\right)
$$

If $D N_{i}$ decides that $P U_{i}$ is active, then $S T_{j}, \forall j \in \mathcal{S}_{i}$ cannot transmit data during the data transmission slot. However, if $D N_{i}$ determines that channel $i$ is idle, then each $S T_{j}, j \in \mathcal{S}_{i}$ has a chance to access the licensed channel $i$ with equal probability. With probability $P_{0 \mid 0, i}$, the secondary data transmission in channel $i$ will be successful. However, with probability $P_{0 \mid 1, i}$, the secondary data transmission in channel $i$ will not be allowed because it interferes with the $P U_{i}$ 's transmission. Therefore, under the hypothesis $\mathcal{H}_{0, i}$, the transmission probability of $S T_{j}, j \in \mathcal{S}_{i}$ is $1 /\left|\mathcal{S}_{i}\right|$, and the transmission time is $T-\delta$. As an example shown in Figure 1(c), $S T_{1}$ and $S T_{2}$ seek to access the licensed channel of $P U_{1}$ (solid arrows), and $S T_{3}$ and $S T_{4}$ seek to access the licensed channel of $\mathrm{PU}_{2}$ (dash arrows).

Given the signal transmit power $P_{t}$, the noise power $\sigma_{n}^{2}$ in channel $i$, and the average channel gain $\left|h_{j, i}\right|$ of the link between the ST-SR pair $j$ in channel $i$, the transmission rate $R_{j, i}$ of $S T_{j}, j \in \mathcal{S}_{i}$ can be modeled as [17]:

$$
R_{j, i}=B \log _{2}\left(1+\left|h_{j, i}\right|^{2} \frac{P_{t}}{\sigma_{n}^{2}}\right) .
$$

Given the CSSA scheme described above, when the sensing duration $\delta$ is fixed, it is a challenge for each SU to decide the channel that it should sense and access. In the next section, we will propose distributed algorithms to solve this spectrum sensing problem using coalitional game theory.

\section{COALITION Formation GAME}

In this section, we formulate the problem of multi-channel energy-efficient cooperative spectrum sensing as a coalition formation game. We propose distributed algorithms to obtain a stable coalition structure that maximizes the aggregate utility of all the coalitions in the system.

Using the terminology of coalitional game theory, we refer to set $\mathcal{S}_{i}$ as coalition $i$. Since there are $M$ licensed channels in the CRN, there are $M$ coalitions in the system, and each SU joins one of those $M$ coalitions. All $S T_{j}, j \in \mathcal{S}_{i}$ would perform cooperative spectrum sensing in channel $i \in \mathcal{M}$ in order to seek the possible transmission opportunities.

The utility function of a coalition is related to both the sensing accuracy and energy efficiency. Specifically, the utility 
function of coalition $\mathcal{S}_{i}, i \in \mathcal{M}$, where $\mathcal{S}_{i} \neq \emptyset$, is given by

$$
U\left(\mathcal{S}_{i}\right)=\frac{P_{0 \mid 0, i}(\delta) \frac{\sum_{j \in \mathcal{S}_{i}} R_{j, i}}{\left|\mathcal{S}_{i}\right|}(T-\delta)-P_{0 \mid 1, i}(\delta) D_{0}\left(1-\frac{\delta}{T}\right)}{P_{s}\left|\mathcal{S}_{i}\right| \delta+P_{0 \mid 0, i}(\delta) P_{t}(T-\delta)},
$$

where $P_{s}$ is the sensing power of $S T_{j}, \forall j \in \mathcal{N}, R_{j, i}$ is the data transmission rate of $S T_{j}, j \in \mathcal{S}_{i}$, and $D_{0}$ is the maximum penalty for interfering with the PU's data transmission. Notice that the penalty term $D_{0}\left(1-\frac{\delta}{T}\right)$ is decreasing with the duration of the sensing slot $\delta$. The denominator in (8) represents the total expected energy consumption in each frame, which is the sum of the expected amount of energy used for sensing (i.e., $\left.P_{s}\left|\mathcal{S}_{i}\right| \delta\right)$ and transmission (i.e., $P_{0 \mid 0, i}(\delta) P_{t}(T-\delta)$ ) in coalition $i$. When $D_{0}$ is set to 0 , the utility function represents the average data rate in coalition $i$ per unit of energy consumed. In other words, it is related to the energy efficiency of the transmission. Also, the utility function takes into account the sensing accuracy by considering the sensing results related to false alarm (i.e., $P_{0 \mid 0, i}(\delta)$ is related to $P_{f, i}(\delta)$ ) and detection (i.e., $P_{0 \mid 1, i}(\delta)$ is related to $\left.P_{d, i}(\delta)\right)$. The value of $U(\emptyset)$ is chosen such that $U\left(\mathcal{S}_{i}\right)>U(\emptyset), \forall \mathcal{S}_{i} \subseteq \mathcal{N}$ and $\mathcal{S}_{i} \neq \emptyset$.

Definition 1: The set $\mathcal{S}=\left\{\mathcal{S}_{1}, \ldots, \mathcal{S}_{M}\right\}$ is a partition of $\mathcal{N}$ if $\mathcal{S}_{i} \cap \mathcal{S}_{l}=\emptyset, \forall i, l \in \mathcal{M}, i \neq l$ and $\bigcup_{i \in \mathcal{M}} \mathcal{S}_{i}=\mathcal{N}$.

Since each of $N$ SUs can join any one of $M$ coalitions, there are $M^{N}$ possible partitions in total. The multi-channel energyefficient cooperative spectrum sensing problem can thus be modelled as a $(\mathcal{N}, U)$ coalition formation game [14]. The goal is to maximize the aggregate utility of the $M$ coalitions in the system. That is, maximize $\sum_{i=1}^{M} U\left(\mathcal{S}_{i}\right)$. In other words, we aim to find a partition $\mathcal{S}^{*}$ which gives the optimal aggregate utility $\sum_{i=1}^{M} U\left(\mathcal{S}_{i}^{*}\right)$.

In order to compare two partitions $\mathcal{S}=\left\{\mathcal{S}_{1}, \ldots, \mathcal{S}_{M}\right\}$ and $\mathcal{T}=\left\{\mathcal{T}_{1}, \ldots, \mathcal{T}_{M}\right\}$, which are partitions of the same set $\mathcal{N}$, we define a relational operator $\triangleright$ for comparison [6], [14].

Definition 2: $\mathcal{S} \triangleright \mathcal{T}$ implies that partition $\mathcal{S}$ is preferred to partition $\mathcal{T}$ in terms of $\sum_{i=1}^{M} U\left(\mathcal{S}_{i}\right)>\sum_{i=1}^{M} U\left(\mathcal{T}_{i}\right)$.

The merge-and-split rule [14] that is commonly used in a coalition formation game (e.g., in [6], [11]) cannot be applied in our game. Since we consider that all the SUs that choose to sense the same channel belong to the same coalition, and each licensed channel is owned by one PU, we cannot either merge two channels into one channel or split one channel into two channels. Therefore, we propose distributed algorithms in order to make sure that the SUs can self-organize in the CRN as time evolves.

In each iteration, Algorithm 1 is executed by $S T_{j}, \forall j \in \mathcal{S}_{i}$ in order to select $D N_{i}$. Since $S T_{j}$ determines which licensed channel $i \in \mathcal{M}$ to sense and access in each frame, therefore $S T_{j}$ knows the value of $i$.

Algorithm 2 is executed by $D N_{i}, \forall i \in \mathcal{M}$ in order to update the partition $\mathcal{S}$ of the $(\mathcal{N}, U)$ coalition formation game. For the $r^{\text {th }}$ iteration in Algorithm 2, all the $D N_{i}^{(r)}, \forall i \in \mathcal{M}$ communicate with each other and select two different channels $m$ and $n$ which satisfy $\left|\mathcal{S}_{m}^{(r)}\right|>0$ and $\left|\mathcal{S}_{n}^{(r)}\right|<N$ (line 4). Then, $D N_{m}^{(r)}$ and $D N_{n}^{(r)}$ will try to update the current
Algorithm 1 Distributed algorithm to select decision node $D N_{i}$, for channel $i \in \mathcal{M}$. The algorithm is executed by $S T_{j}, \forall j \in \mathcal{S}_{i}$.

1: for iteration $r:=1$ to $M A X$ do

2: $\quad S T_{j}$ broadcasts its SNR information $\gamma_{i, j}^{(r)}$ to other secondary transmitters $S T_{k}, \forall k \in \mathcal{S}_{i}^{(r)} \backslash\{j\}$

3: $S T_{j}$ receives the SNR information $\gamma_{i, k}^{(r)}$ from other $S T_{k}, \forall k \in \mathcal{S}_{i}^{(r)} \backslash\{j\}$

4: $\quad q:=\arg \max _{p \in \mathcal{S}_{i}^{(r)}} \gamma_{i, p}^{(r)}$

5: $\quad D N_{i}^{(r)}:=S T_{q}$

6: end for

partition. If $D N_{i}^{(r)}, \forall i \in \mathcal{M}$ is selected as $D N_{m}^{(r)}$, it will execute line 6-11. If $D N_{i}^{(r)}, \forall i \in \mathcal{M}$ is selected as $D N_{n}^{(r)}$, it will execute line 14-21. $S T_{k}, k \in \mathcal{S}_{m}^{(r)}$ is selected (line 4) and is responsible for passing the information of coalition $\mathcal{S}_{m}^{(r)}$ and $U_{m}$ (line 8) to coalition $\mathcal{S}_{n}^{(r)}$ (line 16). The partition for the $(\mathcal{N}, U)$ coalition formation game will be updated by removing $S T_{k}$ from coalition $\mathcal{S}_{m}^{(r)}$ (line 7) and adding $S T_{k}$ to coalition $\mathcal{S}_{n}^{(r)}$ (line 15). After finishing the update, $D N_{n}^{(r)}$ computes the utilities of the newly formed coalitions $\mathcal{S}_{m}^{(r)}$ and $\mathcal{S}_{n}^{(r)}$ (line 17). If the total utility of these two coalitions is increased in the $r^{\text {th }}$ iteration, the newly formed coalitions $\mathcal{S}_{m}^{(r)}$ and $\mathcal{S}_{n}^{(r)}$ will keep this update and $D N_{n}^{(r)}$ will not send the feedback signal to $D N_{m}^{(r)}$; otherwise, $D N_{n}^{(r)}$ will send the feedback signal flag $=1$ (line 19), and $S T_{k}$ will be removed from $\mathcal{S}_{n}^{(r)}$ (line 20) and move back to $\mathcal{S}_{m}^{(r)}$ (line 10).

Given the proposed algorithms, we can prove analytically that Algorithm 2 terminates at a stable partition that achieves the optimal aggregate utility of the system.

Definition 3: A partition $\mathcal{S}=\left\{\mathcal{S}_{1}, \ldots, \mathcal{S}_{M}\right\}$ for the $(\mathcal{N}, U)$ coalition formation game is a stable partition [14], if $\mathcal{S}_{m}$ $\left(\forall m \in \mathcal{M}\right.$ and $\left.\left|\mathcal{S}_{m}\right|>0\right)$ does not have an interest in changing the current partition $\mathcal{S}$ by moving $S T_{k}\left(\forall k \in \mathcal{S}_{m}\right)$ to another coalition $\mathcal{S}_{n}\left(\forall n \in \mathcal{M}, n \neq m\right.$ and $\left.\left|\mathcal{S}_{n}\right|<N\right)$, as defined in Algorithm 2.

Theorem 1: Algorithm 2 terminates at a stable partition that achieves the optimal aggregate utility of the system.

Proof: We first prove that Algorithm 2 terminates at a stable partition. In Algorithm 2, only two coalitions $\mathcal{S}_{m}^{(r)}$ and $\mathcal{S}_{n}^{(r)}$ may change their utilities in the $r^{\text {th }}$ iteration $(r=\{1, \ldots, M A X\})$, and the current partition $\mathcal{S}^{(r)}=$ $\left\{\mathcal{S}_{1}^{(r)}, \ldots, \mathcal{S}_{M}^{(r)}\right\}$ will be updated only if the total utility of coalitions $\mathcal{S}_{m}^{(r)}$ and $\mathcal{S}_{n}^{(r)}$ can be increased by moving $S T_{k}, k \in$ $\mathcal{S}_{m}^{(r)}$ from $\mathcal{S}_{m}^{(r)}$ to $\mathcal{S}_{n}^{(r)}$. Since the utilities of the other $M-2$ coalitions remain the same, the aggregate utility of the system $\sum_{i=1}^{M} U\left(\mathcal{S}_{i}^{(r)}\right)$ of the $r^{\text {th }}$ iteration will either remain the same or increase, compared with $\sum_{i=1}^{M} U\left(\mathcal{S}_{i}^{(r-1)}\right)$. Therefore, every iteration of Algorithm 2 produces a sequence of partitions $\mathcal{S}^{(0)}, \mathcal{S}^{(1)}, \mathcal{S}^{(2)}, \ldots$ with either $\mathcal{S}^{(r)} \triangleright \mathcal{S}^{(r-1)}$ or $\mathcal{S}^{(r)}=$ $\mathcal{S}^{(r-1)}$ for all $r=\{1, \ldots, M A X\}$. However, the number of different partitions for the $(\mathcal{N}, U)$ coalition formation game is 


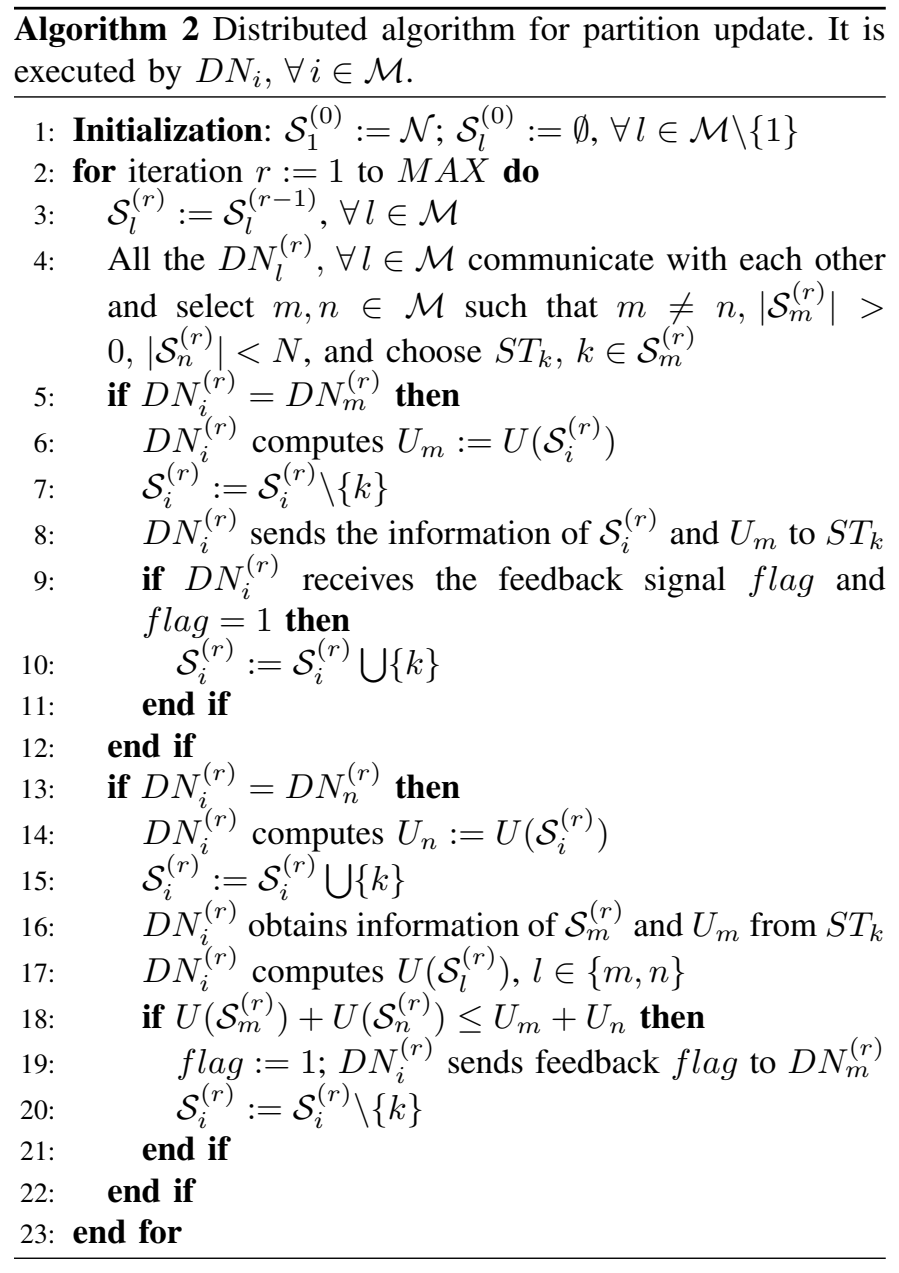

$M^{N}$, which is finite. Therefore, as time evolves, this sequence of partitions will terminate at a partition $\mathcal{S}^{*}=\left\{\mathcal{S}_{1}^{*}, \ldots, \mathcal{S}_{M}^{*}\right\}$, where any non-empty coalition $\mathcal{S}_{m}^{*}, m \in \mathcal{M}$ can never increase the aggregate utility of the system by moving $S T_{k}$ $\left(\forall k \in \mathcal{S}_{m}^{*}\right)$ to any other coalition $\mathcal{S}_{n}^{*}, n \in \mathcal{M}, n \neq m$. According to Definition $3, \mathcal{S}^{*}=\left\{\mathcal{S}_{1}^{*}, \ldots, \mathcal{S}_{M}^{*}\right\}$ is a stable partition for the $(\mathcal{N}, U)$ coalition formation game.

Now we prove that the optimal aggregate utility of the system can be achieved at the stable partition. The aggregate utility $\sum_{i=1}^{M} U\left(\mathcal{S}_{i}\right)$ is a function of partition $\mathcal{S}$. Since the number of different partitions for the $(\mathcal{N}, U)$ coalition formation game is finite, the number of different aggregate utilities of the system is also finite. According to Algorithm 2, we have $\sum_{i=1}^{M} U\left(\mathcal{S}_{i}^{(r)}\right) \geq \sum_{i=1}^{M} U\left(\mathcal{S}_{i}^{(r-1)}\right)$ for all $r=$ $\{1, \ldots, M A X\}$. At the stable partition $\mathcal{S}^{*}=\left\{\mathcal{S}_{1}^{*}, \ldots, \mathcal{S}_{M}^{*}\right\}$, the aggregate utility of the system cannot be increased by updating the partition anymore, therefore, the aggregate utility given by the stable partition $\mathcal{S}^{*}=\left\{\mathcal{S}_{1}^{*}, \ldots, \mathcal{S}_{M}^{*}\right\}$ is the optimal aggregate utility of the system.

\section{Performance Evaluation}

In this section, we first present the results for the convergence and optimality of our proposed distributed algorithms. We then evaluate the performance gain of our proposed CSSA

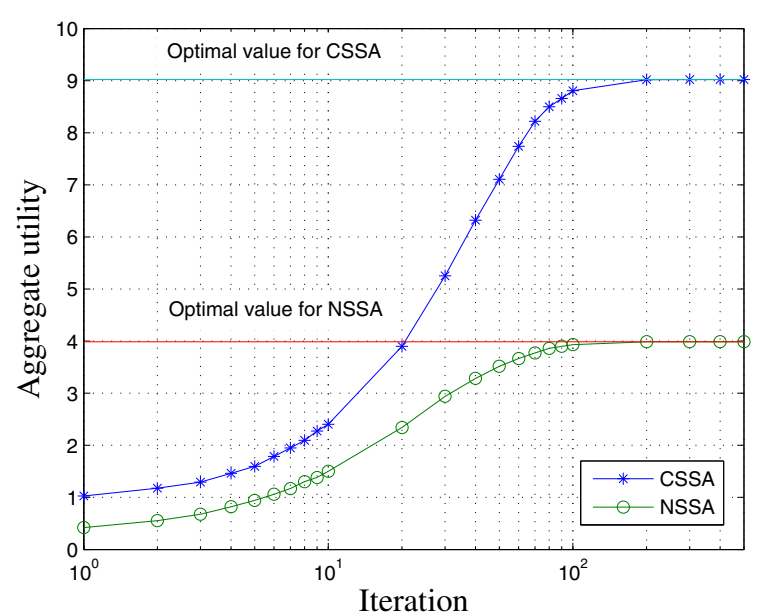

Fig. 2. The aggregate utility of the system versus iterations $(N=10$, $\left.P_{\mathcal{H}_{1}}=0.8\right)$.

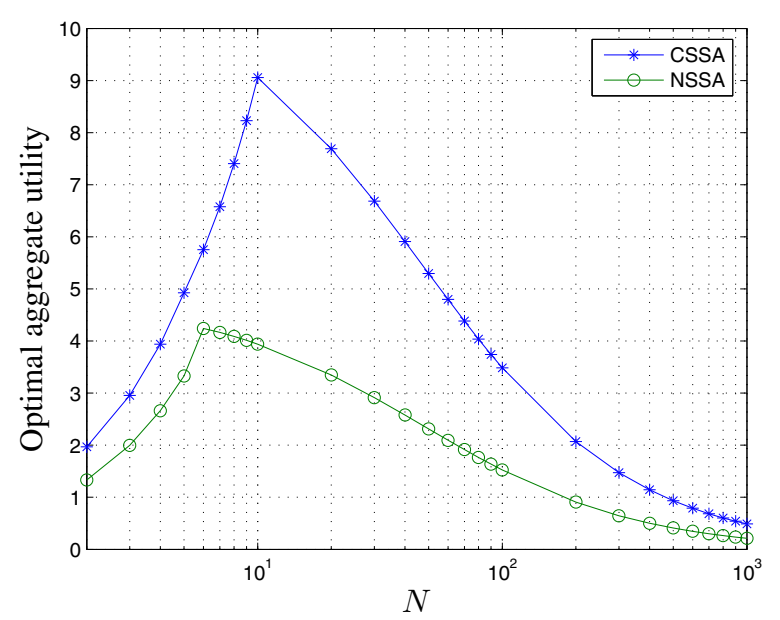

Fig. 3. The optimal aggregate utility of the system versus the number of SUs $N\left(P_{\mathcal{H}_{1}}=0.8\right)$.

scheme over the noncooperative spectrum sensing and accessing (NSSA) scheme under different system parameters using MATLAB. As for the NSSA scheme, the SUs perform local spectrum sensing instead of cooperative spectrum sensing. In the simulation, we consider that there are five PUs $(M=5)$ and ten ST-SR pairs $(N=10)$. The bandwidth of each primary licensed channel is $B=10 \mathrm{kHz}$. Other parameters used in our simulation are set as follows: the length of each time frame $T=100 \mathrm{~ms}$; the sampling frequency $f_{s}=1 \mathrm{kHz}$; the transmit power of each ST $P_{t}=10 \mathrm{~mW}$; the sensing power of each ST $P_{s}=10 \mathrm{~mW}$; the detection threshold $\varepsilon=0.2 \mathrm{~mW}$; the noise power $\sigma_{n}^{2}=0.1 \mathrm{~mW}$. The probability that $P U_{i}, i \in \mathcal{M}$ is active is $P_{\mathcal{H}_{1, i}}=P_{\mathcal{H}_{1}}=0.8, \forall i \in \mathcal{M}$. We choose the sensing duration $\delta=5 \mathrm{~ms}$ and the penalty term $D_{0}=2$. Each point is averaged over 100 independent simulation runs.

We compare the performance of our proposed CSSA scheme with the NSSA scheme. Figure 2 shows the aggregate utility in each iteration using the proposed algorithms. We can see that the aggregate utility of the $M$ coalitions in the system under CSSA is higher than that under NSSA. Our proposed algorithms result in the self-organization of the SUs that 


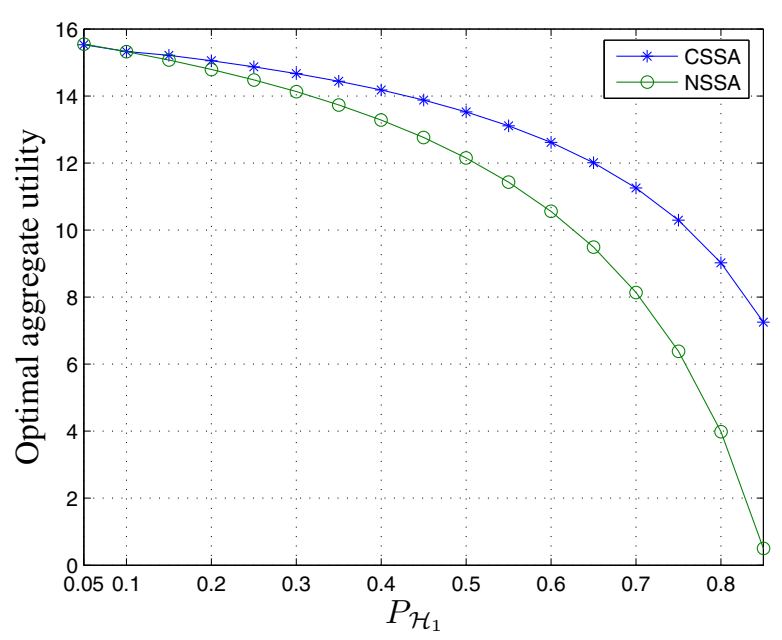

Fig. 4. The optimal aggregate utility of the system versus the probability that the PUs are active $P_{\mathcal{H}_{1}}(N=10)$.

achieves a higher aggregate utility after each iteration, and eventually reaches a stable partition after about 200 iterations. Furthermore, the aggregate utility of the stable partition obtained by our distributed algorithms is the same as the optimal aggregate utility obtained by a centralized algorithm.

In Figure 3, we show the optimal aggregate utility of the system obtained by the proposed algorithms versus the number of SUs $N$ in the $\mathrm{CRN}$ when $P_{\mathcal{H}_{1}}=0.8$. Our results show that the performance of CSSA is better than NSSA. For both schemes, the optimal aggregate utility of the system first increases with $N$, and then decreases with $N$ after reaching the maximum point. The reason is that when $N$ is small, increasing the number of SUs improves the spectrum utilization. Although the energy consumption of the system is increased when more SUs sense and transmit in the CRN, the average secondary data rate is increased more significantly due to the improved sensing accuracy. If $N$ is increased further, the energy consumption for spectrum sensing increases linearly, but the sensing accuracy is not improved as significantly. Thus, the optimal aggregate utility is reduced.

In Figure 4, we show the optimal aggregate utility of the system obtained by the proposed algorithms versus the probability that the PUs are active $P_{\mathcal{H}_{1}}$ when $N=10$. Our results show that the performance of CSSA is better than NSSA. When $P_{\mathcal{H}_{1}}$ is small, the optimal aggregate utility of CSSA is slightly higher than that of NSSA. The performance gap increases with $P_{\mathcal{H}_{1}}$ because the improvement of sensing accuracy with CSSA over NSSA is more significant when the PU has a higher traffic load.

\section{CONCLUSIONS}

In this paper, we studied energy-efficient spectrum sensing in a CRN with multiple licensed channels and multiple SUs. We proposed a CSSA scheme and formulated the multichannel spectrum sensing problem as a coalition formation game. The utility function of each coalition takes into account both the sensing accuracy and energy efficiency. Since all the SUs that choose to sense the same channel belong to the same coalition, the commonly used merge-and-split rule cannot be applied in our coalition formation game. Therefore, we proposed distributed algorithms to find the stable partition that achieves the optimal aggregate utility of the system distributively for the CSSA scheme. Simulation results showed that the performance of our proposed CSSA scheme is better than the NSSA scheme. Besides, the results showed that our proposed distributed algorithms result in the self-organization of the SUs that achieves a higher aggregate utility after every iteration, and that there is a stable partition for our formulated coalition formation game.

\section{ACKNOWLEDGMENT}

This research is supported by the Natural Sciences and Engineering Research Council (NSERC) of Canada.

\section{REFERENCES}

[1] "Spectrum policy task force," Federal Communications Commission, Tech. Rep., 2002.

[2] J. Mitola III and G. Q. Maguire Jr., "Cognitive radio: Making software radios more personal," IEEE Personal Communications, vol. 6, no. 4, pp. 13-18, Aug. 1999.

[3] I. F. Akyildiz, B. F. Lo, and R. Balakrishnan, "Cooperative spectrum sensing in cognitive radio networks: A survey," Elsevier Physical Communication, vol. 4, no. 1, pp. 40-62, March 2011.

[4] M. J. Marcus, "Unlicensed cognitive sharing of TV spectrum: The controversy at the Federal Communications Commission," IEEE Communications Magazine, vol. 43, no. 5, pp. 24-25, May 2005.

[5] Y.-C. Liang, Y. Zeng, E. C. Peh, and A. T. Hoang, "Sensing-throughput tradeoff for cognitive radio networks," IEEE Trans. on Wireless Communications, vol. 7, no. 4, pp. 1326-1337, April 2008.

[6] W. Saad, Z. Han, T. Basar, M. Debbah, and A. Hjorungnes, "Coalition formation games for collaborative spectrum sensing," IEEE Trans. on Vehicular Technology, vol. 60, no. 1, pp. 276-297, Jan. 2011.

[7] H. Su and X. Zhang, "Energy-efficient spectrum sensing for cognitive radio networks," in Proc. of IEEE ICC, Cape Town, South Africa, May 2010.

[8] C. Song and Q. Zhang, "Cooperative spectrum sensing with multichannel coordination in cognitive radio networks," in Proc. of IEEE ICC, Cape Town, South Africa, May 2010.

[9] Q. Zhao, S. Geirhofer, L. Tong, and B. M. Sadler, "Opportunistic spectrum access via periodic channel sensing," IEEE Trans. on Signal Processing, vol. 56, no. 2, pp. 785-796, Feb. 2008.

[10] W.-Y. Lee and I. F. Akyildiz, "Optimal spectrum sensing framework for cognitive radio networks," IEEE Trans. on Wireless Communications, vol. 7, no. 10 , pp. 3845-3857, Oct. 2008.

[11] W. Wang, B. Kasiri, J. Cai, and A. S. Alfa, "Distributed cooperative multi-channel spectrum sensing based on dynamic coalitional game," in Proc. of IEEE Globecom, Miami, FL, Dec. 2010.

[12] M. Gandetto and C. Regazzoni, "Spectrum sensing: A distributed approach for cognitive terminals," IEEE Journal on Selected Areas in Communications, vol. 25, no. 3, pp. 546-557, April 2007.

[13] T. C. Aysal, S. Kandeepan, and R. Piesiewicz, "Cooperative spectrum sensing with noisy hard decision transmissions," in Proc. of IEEE ICC, Dresden, Germany, June 2009.

[14] W. Saad, Z. Han, M. Debbah, A. Hjorungnes, and T. Basar, "Coalitional game theory for communication networks," IEEE Signal Processing Magazine, vol. 26, no. 5, pp. 77-97, Sept. 2009.

[15] W. Zhang and K. B. Letaief, "Cooperative spectrum sensing with transmit and relay diversity in cognitive radio networks," IEEE Trans. on Wireless Communications, vol. 7, no. 12, pp. 4761-4766, Dec. 2008.

[16] G. Ganesan and Y. Li, "Agility improvement through cooperative diversity in cognitive radio," in Proc. of IEEE Globecom, St. Louis, MO, Dec. 2005.

[17] O. Simeone, I. Stanojev, S. Savazzi, Y. Bar-Ness, U. Spagnolini, and R. Pickholtz, "Spectrum leasing to cooperating secondary ad hoc networks," IEEE Journal on Selected Areas in Communications, vol. 26, no. 1, pp. 203-213, Jan. 2008. 